\title{
Diagnosing human African trypanosomiasis in Angola using a card agglutination test: observational study of active and passive case finding strategies
}

Walter O Inojosa, Inacio Augusto, Zeno Bisoffi, Teofile Josenado, Paolo M Abel, August Stich, Christopher J M Whitty

\begin{abstract}
Objective To assess the operational feasibility of detecting human African trypanosomiasis by active and passive case finding using the card agglutination test with serial dilution of serum to guide treatment.

Setting Trypanosomiasis control programme in the Negage focus, northern Angola, during a period of civil war.

Design Observational study.

Participants 359 patients presenting themselves to health centres with symptoms (passive case finding) and 14446 people actively screened in villages.

Main outcome measures Whole blood and serological tests at different dilutions using the card agglutination test, and detection of parasites by microscopy.

Results Active case finding identified 251 people with a positive card agglutination test result, 10 of whom had confirmed parasites. In those presenting for investigation 34 of 51 with a positive card agglutination test result at the dilution of $1: 8$ or more used to guide treatment had parasites in blood, lymph node fluid, or cerebrospinal fluid, compared with 10 of 76 in those detected by active case finding: positive predictive values of $67 \%$ for passive case detection and 13\% for active case detection. Only at a cut-off dilution more than 1:32 was the positive predictive value in active case detection reasonable $(46 \%)$ and at this dilution $40 \%$ of microscopically proved cases were missed.

Conclusions The card agglutination test is useful for initial screening in active detection of cases with human African trypanosomiasis but, given the toxicity of the drugs, serology using the card agglutination test should be not used alone to guide treatment after active case finding. A second confirmatory test is needed.
\end{abstract}

\section{Introduction}

Human African trypanosomiasis is one of the major parasitic diseases of humans and is increasing in many parts of the continent. ${ }^{1}$ The disease, caused by protozoans transmitted by the tsetse fly, has two forms, Trypanosoma brucei gambiense and $T b$ rhodesiense. $T \quad b$ gambiense causes most of the estimated $300000-500000$ infections a year. The parasites are found in the blood and lymphatic system but in more advanced disease invade the brain. The disease is most common in areas of current or recent civil breakdown; the Democratic Republic of Congo has the greatest number of cases, followed by Angola with around 100000 , but as only a fraction of the exposed population is under adequate surveillance these are estimates. $T$ b gambiense causes sleeping sickness, the chronic, slowly progressive form of human African trypanosomiasis, which is mostly fatal if left untreated.

Early diagnosis and treatment of trypanosomiasis is essential, as treatment of late stage disease is associated with high mortality. Humans are the reservoir of infection, so early treatment helps to interrupt the parasite's transmission cycle, providing an efficient control strategy for active case finding campaigns. ${ }^{2}$ At the same time over-diagnosis and consequent over-treatment are potentially dangerous for patients. The most commonly used drug for second stage treatment is melarsoprol, which contains arsenic. The drug is highly toxic, with $2 \%$ to $10 \%$ mortality during or shortly after treatment, mostly from arsenical reactive encephalopathy. ${ }^{3}$ Attempts to modify the drug's schedule have not reduced mortality. ${ }^{4}$ A newer drug, eflornithine, is probably safer than melarsoprol, ${ }^{5}$ but because of costs and difficulties of administration it has not been adopted as first line treatment of second stage disease in most sub-Saharan countries. First stage treatment with pentamidine is less toxic but can cause severe side effects, and up to $1 \%$ mortality has been reported during treatment. ${ }^{6}$ Diagnostic systems therefore need to detect cases early and minimise false positives to avoid exposing patients to drugs that are dangerous or expensive, or both; a combination that is difficult to achieve.

Even in high resource settings diagnosing trypanosomiasis is not easy. ${ }^{7}$ Classically, diagnosis is based on direct visualisation of parasites in the blood, lymph node fluid, or cerebrospinal fluid. ${ }^{8}$ Sensitivity of microscopy is often limited owing to low or fluctuating parasitaemia. Examination of wet preparations of lymph node fluid can detect 30\%-70\% of cases, and examination of blood even by the most sensitive concentration technique of mini-anion exchange centrifugation only adds another 5\%-10\% of cases. ${ }^{9}$ Routine lumbar puncture is not recommended in asymptomatic people with negative haemolymphatic examination results. Consequently at least $20 \%$ of infected people are lost during active case finding and treatment surveys using conventional methods of diagnosis.

The card agglutination test for trypanosomiasis, a method developed for the detection of $T$ b gambiense specific antibodies, ${ }^{10}$ is a simple tool for rapid screening of high patient numbers under field conditions using whole blood. Sensitivity for $T \mathrm{~b} \mathrm{gam}$ biense varies between $87 \%$ and $98 \%$, with an average of $95 \%,{ }^{11}$ although this may vary by setting. ${ }^{12}$ The test is not sensitive for $T$ $b$ rhodesiense. The test kits are cheap (around 30p) and screening using the test is considered more cost effective then conventional screening of lymph nodes. ${ }^{13}$ The World Health Organization rec- 
ommends follow-up every 3-6 months for people who have a positive test result but no parasites on microscopic examination of blood and lymph node fluid. This is difficult to maintain in areas of complex emergency, however, where much of the trypanosomiasis burden is found. Serial dilution of serum before using the card agglutination test has been suggested as a diagnostic confirmatory assay in those who have an initial positive result on screening to avoid the need for a prolonged and potentially fatal wait. ${ }^{14}$ As with other serological tests, diluting serum increases the specificity at the possible expense of sensitivity; the higher the dilution at which a test remains positive the more likely it is to be a true positive.

During the recent civil war in Angola few areas of the country could be reached easily and, as in many endemic areas where trypanosomiasis is common, a screening approach requiring only one visit was the only realistic way to identify cases and to contain the infection reservoir using available resources. Between 1999 and 2002 the control programme for trypanosomiasis in Angola decided that all people who had a positive serology result at a serum dilution of 1:8 or more with the card agglutination test should be considered infected and therefore treated regardless of evidence of parasites on microscopy. ${ }^{15}$

The empirical decision to treat a patient with no parasites detected on microscopy on the basis of a high titre on the card agglutination test has still to be fully evaluated under field conditions. Although this decision aims at a major increase in detection of true cases it could lead to over-diagnosis and subsequent treatment of false positives, which is expensive and potentially hazardous. We determined whether titration using the card agglutination test can be used to guide treatment in potential cases of human African trypanosomiasis detected by passive and active case finding.

\section{Participants and methods}

The study took place from February to May 2001 in the Negage focus of the province of Uíge in northern Angola, where the non-governmental organisation Caritas runs the human African trypanosomiasis control programme, Angotrip..$^{16}$ The study took place during the final stage of the civil war. Patients with symptoms presented themselves to the specialist treatment centre in Negage (passive case finding). Common symptoms were headache, sleep disorder, arthralgia, itching, symptomatic lymphadenopathy, behavioural change, and neurological problems (especially ataxia). Active case finding was by screening people of all ages for trypanosomiasis in adjacent villages, irrespective of symptoms, using village lists drawn up by municipal authorities. All villagers were asked to attend screening at the local school; $63 \%$ of those identified on the municipal lists attended, although as these lists are often outdated the true proportion of the population screened was probably much higher.

We consecutively registered all patients from both groups and screened whole blood from each by the conventional card agglutination test (Laboratory of Serology, Institute of Tropical Medicine, Antwerp, Belgium). We considered asymptomatic people with a negative test result free of the disease and they had no further investigations. In accordance with the diagnostic protocol of the national control programme (figure), ${ }^{15}$ people with a positive test result underwent cervical lymph node examination for lymphadenopathy and, if present, subsequent examination of a wet preparation of lymph node fluid. This examination was carried out by conventional methods as recommended by WHO. ${ }^{17}$ If trypanosomes were found the patient was treated. Those without palpable lymph nodes or with a negative lymph

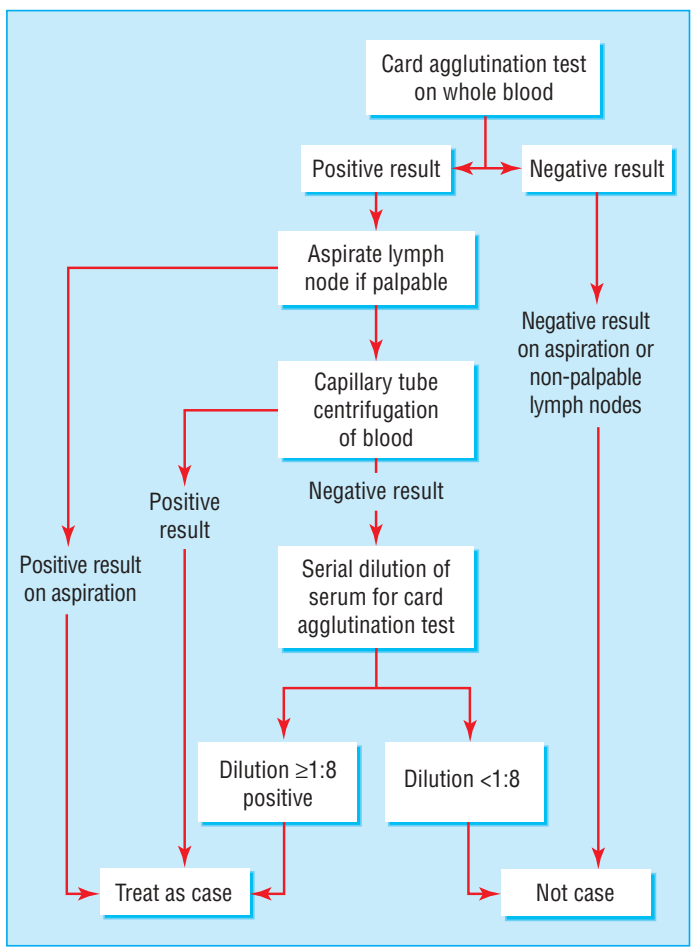

Flow of screening process

node fluid result had their blood examined for parasites by capillary tube centrifugation (two tubes for each person) ${ }^{18}$; again the patient was treated if trypanosomes were found. The cerebrospinal fluid was double centrifuged and searched for trypanosomes. ${ }^{19}$ We used the Fuchs-Rosenthal chamber to count white blood cells in the cerebrospinal fluid.

For people with a positive card agglutination test result but negative microscopic examination of blood and lymph node fluid, we determined the serum end titre using the test. In addition, after obtaining informed consent, patients with confirmed presence of parasites had a further blood sample taken to determine their serological status. For serum titration we centrifuged $5 \mathrm{ml}$ of blood collected by venepuncture; we tested serum with serial twofold dilution in card agglutination test buffer solution and determined the highest dilution at which a sample remained positive. Following the national guidelines at the time we considered people infected who were either positive for parasites or positive using the card agglutination test result at a serum dilution of 1:8 or more. They underwent a lumbar puncture for differentiation of parasite stage, with cerebrospinal fluid searched for trypanosomes and a white blood cell count carried out. Finally, they were treated with pentamidine or melarsoprol according to the parasite stage.

We entered laboratory and clinical data into Access (Microsoft 97) and analysed the data using Stata version 8. Evaluation of card agglutination test titres was carried out by classic $2 \times 2$ tables. We assessed the sensitivity and positive predictive value of serum titres at various cut-off dilutions. The gold standard was microscopically proved cases. Microscopy is $100 \%$ specific, although it has a recognised false negative rate. Absolute sensitivities and specificities in the general population cannot be calculated because for practical and ethical reasons the full parasitological investigation was not carried out on all those with a negative card agglutination test result on whole blood, but we report the relative sensitivities of higher dilutions. 
Table 1 Evaluation of card agglutination test compared with parasite proved disease after active case finding of people with human African trypanosomiasis identified by mass screening

\begin{tabular}{|c|c|c|c|}
\hline \multirow[b]{2}{*}{$\begin{array}{l}\text { Cut-off dilution using } \\
\text { card agglutination test }\end{array}$} & \multicolumn{2}{|c|}{ Conventional parasitological methods* } & \multirow[b]{2}{*}{ Total } \\
\hline & $\begin{array}{c}\text { No with positive } \\
\text { result }\end{array}$ & $\begin{array}{c}\text { No with negative } \\
\text { result }\end{array}$ & \\
\hline \multicolumn{4}{|l|}{ Whole blood: } \\
\hline Positive result & 10 & $241 \dagger$ & 251 \\
\hline Negative result & 0 & 14195 & 14195 \\
\hline Total & 10 & 14436 & 14446 \\
\hline \multicolumn{4}{|l|}{$\geq 1: 8:$} \\
\hline Positive result & 10 & $66 \dagger$ & 76 \\
\hline Negative result & 0 & 14370 & 14370 \\
\hline Total & 10 & 14436 & 14446 \\
\hline \multicolumn{4}{|l|}{$\geq 1: 16:$} \\
\hline Positive result & 10 & $39 \dagger$ & 49 \\
\hline Negative result & 0 & 14397 & 14397 \\
\hline Total & 10 & 14436 & 14446 \\
\hline \multicolumn{4}{|l|}{$\geq 1: 32$} \\
\hline Positive result & 6 & $7 \dagger$ & 13 \\
\hline Negative result & 4 & 14429 & 14433 \\
\hline Total & 10 & 14436 & 14446 \\
\hline
\end{tabular}

${ }^{*}$ Capillary tube centrifugation, wet examination of lymph fluid, and examination of cerebrospinal fluid.

†False positive result for serology.

\section{Results}

We consecutively screened 14446 people of all age groups by active case finding in the villages. In total 359 people with symptoms presented themselves to the treatment centre for examination and were consecutively registered in the study. They constituted patients detected by passive case finding.

Active case finding in the villages led to the identification of 251 people with a positive card agglutination test result (seroprevalence 1.7\%). Seventy six people aged between 5 years and 72 years (mean 33.2 years) had a positive reaction in serum titration of $1: 8$ or more, but in only $10(13 \%)$ people were parasites detected in either lymph node fluid or blood (table 1). Of these just over half had symptoms, mostly non-specific: 43 had headaches, 21 had itching, 10 had sleep disorders, 13 had lymphadenopathy, and 4 had behavioural change or neurological symptoms. Parasites were found only in people with card agglutination test titres of 1:16 or more. No patients identified by active case finding had detectable parasites in the cerebrospinal fluid. The prevalence of parasitologically proved disease was low: $0.07 \%$ (10 of 14446 people).

Eighty three people identified by passive case finding had a positive card agglutination test result (seroprevalence 23.1\%). Fifty one people aged between 1 year and 56 years (mean 21.2 years) had elevated card agglutination test titres, of whom 34 $(67 \%)$ had detectable parasites in lymph node fluid, blood, or cerebrospinal fluid (table 2). Parasites were not found in people with card agglutination test titres less than $1: 8$, and only one patient with parasitologically proved disease had a titre equal to 1:8. Table 3 shows the distribution of parasites into blood, lymph nodes, and cerebrospinal fluid. Haemolymphatic examination detected $65 \%(22 / 34)$ of patients with parasitologically proved disease. In 12 patients (35\% of the total) trypanosomes were found only in the cerebrospinal fluid; of these, six were either asymptomatic or had non-specific symptoms not suggesting a neurological diagnosis.

Table 4 shows the sensitivity and positive predictive values of serology by active and passive case finding strategies. A positive card agglutination test result on whole blood using active case
Table 2 Evaluation of card agglutination test compared with parasite proved disease using passive case detection where patients have presented with symptoms of trypanosomiasis for diagnosis

\begin{tabular}{|c|c|c|c|}
\hline \multirow{2}{*}{$\begin{array}{l}\text { Cut-off dilution using } \\
\text { card agglutination test }\end{array}$} & \multicolumn{2}{|c|}{ Conventional parasitological methods* } & \multirow[b]{2}{*}{ Total } \\
\hline & $\begin{array}{l}\text { No with positive } \\
\text { result }\end{array}$ & $\begin{array}{c}\text { No with negative } \\
\text { result }\end{array}$ & \\
\hline \multicolumn{4}{|l|}{ Whole blood: } \\
\hline Positive result & 51 & $32 \dagger$ & 83 \\
\hline Negative result & 0 & 276 & 276 \\
\hline Total & 51 & 308 & 359 \\
\hline \multicolumn{4}{|l|}{$\geq 1: 8:$} \\
\hline Positive result & 34 & $17 \dagger$ & 51 \\
\hline Negative result & 0 & 308 & 308 \\
\hline Total & 34 & 325 & 359 \\
\hline \multicolumn{4}{|l|}{$\geq 1: 16:$} \\
\hline Positive result & 33 & $11 \dagger$ & 44 \\
\hline Negative result & 1 & 314 & 315 \\
\hline Total & 34 & 325 & 359 \\
\hline \multicolumn{4}{|l|}{$\geq 1: 32:$} \\
\hline Positive result & 21 & $2 \dagger$ & 23 \\
\hline Negative result & 13 & 323 & 336 \\
\hline Total & 34 & 325 & 359 \\
\hline
\end{tabular}

${ }^{*}$ Capillary tube centrifugation, wet examination of lymph fluid, and examination of cerebrospinal fluid.

†False positive result for serology.

finding has a $4 \%$ positive predictive value with a ratio of false to true positives of 24:1. When the cut-off titre is $1: 8$, the current threshold to treat, the positive predictive value is $13.2 \%$. The effect of further serum dilution cut-off points are also shown in table 4. At a cut-off dilution greater than 1:32 the positive predictive value improves to $46 \%$, but $40 \%$ of microscopically proved cases are missed.

When using a passive case finding strategy with self referring patients, the card agglutination test on whole blood has a $61.4 \%$ positive predictive value. When the cut-off dilution is $1: 8$, the positive predictive value is $66.7 \%$. When the cut-off dilution is $1: 32$, the positive predictive value rises to $91.3 \%$, but $38 \%$ of parasitologically proved cases are missed.

Table 3 Body fluids in which trypanosomes were found by passive case detection

Body fluid No $(\%)$ of participants $(\mathrm{n}=34)$

Lymph fluid $6(18)$

Lymph fluid and cerebrospinal fluid

5 (15)

Blood

8 (24)

Blood and cerebrospinal fluid

$3(9)$

Cerebrospinal fluid

$12(35)$

Table 4 Relative sensitivity and positive and negative predictive values of card agglutination test compared with parasitologically proved trypanosomiasis by active and passive case detection

\begin{tabular}{|c|c|c|}
\hline $\begin{array}{l}\text { Cut-off dilution using card } \\
\text { agglutination test }\end{array}$ & Relative sensitivity (\%)* & $\begin{array}{l}\% \text { positive predictive } \\
\text { value }(95 \% \mathrm{Cl})\end{array}$ \\
\hline \multicolumn{3}{|l|}{ Active case detection: } \\
\hline Whole blood & - & 4.0 (2 to 7$)$ \\
\hline$\geq 1: 8$ & 100 (66 to 100$)$ & 13.2 (7 to 23$)$ \\
\hline$\geq 1: 16$ & 100 (66 to 100) & 20.4 (11 to 35$)$ \\
\hline$\geq 1: 32$ & 60.1 (27 to 86 ) & 46.2 (20 to 74$)$ \\
\hline \multicolumn{3}{|l|}{ Passive case detection: } \\
\hline Whole blood & 100 (91 to 100) & 61.4 (50 to 72$)$ \\
\hline$\geq 1: 8$ & 100 (87 to 100) & 66.7 (52 to 79$)$ \\
\hline$\geq 1: 16$ & 97.1 (83 to 100) & 75.0 (59 to 86$)$ \\
\hline$\geq 1: 32$ & 61.8 (44 to 77$)$ & 91.3 (70 to 98$)$ \\
\hline
\end{tabular}

*Starting population in active case finding of those screening positive on whole blood. 


\section{Discussion}

At the prevalence of human African trypanosomiasis found in the general population $(<1 \%)$ the positive predictive value of the card agglutination test at the conventional serological cut-off dilution of 1:8 is too low (13\%) to guide treatment on its own. Given the cost and significant toxicity of the drugs used in the treatment of trypanosomiasis this is a serious problem; where positive serological tests alone are used to guide treatment (as had to be done in Angola at the time of our study) the overall effect could even be harmful. In our setting the overall incidence of reactive arsenical encephalopathy in those treated with melarsoprol was $7.5 \%$. The specificity of the card agglutination test is considerably less than $100 \%$, probably because other diseases that induce false positive reactions (specifically in the area: malaria, schistosomiasis, and filariasis) may cause symptoms leading people to present themselves for diagnosis. For any test even high specificities will correspond to low positive predictive values when the prevalence of disease is low. According to our data, when the prevalence of human African trypanosomiasis is about $10 \%$, only a cut-off dilution of $1: 32$ in passive surveillance presents reasonable high positive predictive value (91\%) to be a sole guide to treatment with the available drugs, but at the cost of missing a significant (and unacceptably high) number of true cases.

Although microscopy is virtually $100 \%$ specific the card agglutination test has a known rate of false positives, and this is higher in endemic countries than when tested against blood from people in non-endemic areas (often used to determine specificities for tests). False positive low titre (1:5) card agglutination test results in people with schistosomiasis, filariasis, or toxoplasmosis have been reported..$^{20}$ The presence of antimalarial antibodies caused by recurrent malaria has been associated with false positive results in the indirect fluorescent antibody test in $5 \%$ of cases. ${ }^{21}$ Both the indirect fluorescent antibody test and the card agglutination test utilise freeze dried Trypanosoma brucei gambiense LiTat 1.3 antigen. Tsetse flies are generally infected by non-pathogenic trypanosomes at higher rates (often more than $10 \%$ ) than by $T b$ gambiense (invariably below $0.5 \%$ ), and both Trypanosoma congolense and Trypanosoma brucei brucei (nonpathogenic) can lead to positive card agglutination test results in infected animals. It can be hypothesised that non-pathogenic trypanosomes might persist even in humans long enough to induce a transiently positive card agglutination test result.

It is possible that newer and more specific serological tests could improve on this, but even the best tests are not $100 \%$ specific under field conditions, which, given the dangers or expense of treatment in human African trypanosomiasis, is a serious problem in settings of low prevalence and poor resources. ${ }^{22}{ }_{23}$ The results of our study suggest that for active case finding of human African trypanosomiasis, serology is likely to be useful in identifying those who need further testing with an unrelated test, currently by conventional parasitological methods, but possibly in the future by a second more specific serological test in much the same way as blood is screened for HIV.

Carrying out research in complex emergency settings such as civil war imposes limitations. The chance is that some, although probably only a few, patients who have a positive card agglutination test result but whose disease is not parasitologically proved would have gone on to have detectable parasites if repeatedly sampled as recommended by WHO. The prevailing situation in Angola at the time of the study did not make this a practical option. Prospective follow-up studies of people with a positive card agglutination test result but who are negative for parasites with periodical parasitological examinations have given varying results: in one study a 12 month follow-up of 71 people initially only positive on the card agglutination test subsequently found 22 cases of infection, ${ }^{17}$ whereas in a 24 month follow-up of 77 people initially exclusively positive at serology subsequently found only one case of infection. ${ }^{24}$

During the Angolan civil war many areas were hardly accessible for health workers. The human African trypanosomiasis control strategy had to be adapted to the reality that a single examination of villagers might be their only chance to access treatment against a fatal disease. Case finding is a key part of control of trypanosomiasis. Even in stable situations long delays occur in diagnosis, during which patients will deteriorate, possibly irreversibly, while remaining a reservoir of infection. ${ }^{25}$ The introduction of titration on the card agglutination test helped to identify many infected patients and possibly to contain the spread of the infection, but also subjected an unknown number of people who were false positive to harmful treatments. Since 2002 Angola has been heading for political stability. Control programmes can now undergo the transition from a humanitarian crisis intervention programme to professional disease containment and surveillance where individual case management can again be given more consideration. Where resources are severely constrained, as they are in most areas where human African trypanosomiasis is a serious problem, using two tests where one is adequate has to be avoided as it is costly. ${ }^{13}$ Our study, however, suggests that the potential dangers of using laboratory investigations that have even a reasonable specificity as the sole guide for trypanosomiasis treatment where the prevalence is low (all active case finding) are considerable in trypanosomiasis. Although serology has a major part to play in active case identification, treatment should be based on a two stage screening process where the first test is followed by another, independent test in those found positive.

Contributors: WOI initiated and designed the study with assistance from ZB, PMA, and AS. WOI, PMA, and IA carried out the study. The data were analysed and written up by WOI and CJMW with contributions from all authors. WOI is guarantor.

Funding: This study was funded by Angotrip, which is supported by the charity Caritas.

Competing interests: None declared.

Ethical approval: This study was approved by Angotrip and the relevant local authorities.

\section{What is already known on this topic}

To prevent potentially fatal progression of human African trypanosomiasis, and for control of the disease, cases must be diagnosed early

Active case detection using the card agglutination test with treatment given to those with a positive test at 1:8 dilution has been advocated

\section{What this study adds}

Using the card agglutination test as the sole guide to treatment of those identified by active case detection leads to substantial over-diagnosis of the disease, with potentially serious consequences

A two stage screening process with a sensitive test for active case detection and a specific test for case confirmation is the most appropriate strategy 
1 Stich A, Abel PM, Krishna S. Human African trypanosomiasis. BMJ 2002;325:203-6.

2 Méda HA, Pépin J. The epidemiology and control of human African trypanosomiasis Adv Parasitol 2001;49:71-132.

3 Pépin J, Milord F. The treatment of human African trypanosomiasis. Adv Parasitol 1994;33:1-47.

4 Schmid C, Nkunku S, Merolle A, Vounatsou P, Burri C. Efficacy of 10-day melarsoprol schedule 2 years after treatment for late-stage gambiense sleeping sickness. Lancet 2004;364:789-90.

5 Van Nieuwenhove S. Present strategies in the treatment of human African trypanosomiasis. In: Dumas M, Bouteille B, Buguet A, eds. Progress in human African trypanosomiasis, sleeping sickness. Paris: Springer, 1999:253-80.

6 Jha TK, Sharma VK. Pentamidine-induced diabetes mellitus. Trans R Soc Trop Med Hyg 1984;78:252-3.

7 Lejon V, Boelaert M, Jannin J, Moore A, Buscher P. The challenge of Trypanosoma brucei gambiense sleeping sickness diagnosis outside Africa. Lancet Infect Dis
2003:3:804-8.

8 Van Meirvenne N. Biological diagnosis of human African trypanosomiasis. In: Dumas M, Bouteille B, Buguet A, eds. Progress in human African trypanosomiasis, sleeping sickness. Paris: Springer, 1999

9 World Health Organization. Control and surveillance of African trypanosomiasis. Report of a WHO expert committee. WHO, Geneva: WHO, technical report series, 1998:881.

10 Magnus E, Vervoot T, Van Meirvenne N. A card-agglutination test with stained trypanosomes (CATT) for the serological diagnosis of $\mathrm{T} b$ gambiense trypanosomiasis. Ann Soc Belg Med Trop 1978:58:169-76.

11 Robays J, Bilengue MM, Van der Stuyft P, Boelaert M. The effectiveness of active population screening and treatment for sleeping sickness control in the Democratic Republic of Congo. Trop Med Int Health 2004;9:542-50.

12 Truc P, Lejon V, Magnus E, Jamonneau V, Nangouma A, Verloo D, et al. Evaluation of the micro-CATT, CATT/Trypanosoma brucei gambiense, and LATEX/T b gambiense methods for serodiagnosis and surveillance of human African trypanosomiasis in wes and central Africa. Bull World Health Organ 2002;80:882-6.

13 Lutumba P, Robays J, Miaka C, Kande V, Simarro PP, Shaw AP, et al. Efficience de différentes stratégies de détection de la trypanosomiase humaine Africaine à T. b. gambiense. Trop Med Int Health 2005;10:347-56.

14 Simarro PP, Ruiz JA, Franco JR, Josenando T. Attitude towards CATT-positive individuals without parasitological confirmation in the African trypanosomiasis $(\mathrm{T} b$ gambiense) focus of Quicama (Angola). Trop Med Int Health 1999;12:858-61

15 Stanghellini A, Josenando T. The situation of sleeping sickness in Angola: a calamity. Stanghellini A, Josenando T. The
Trop Med Int Health 2001;6:330-4.

16 Abel PM, Kiala G, Loa V, Behrend M, Musolf J, Fleischmann H, et al. Retaking sleeping sickness control in Angola. Trop Med Int Health 2004;9:141-8

17 World Health Organization. Trypanosomiasis control manual. Geneva: WHO, 1983

18 Woo PTK. The haematocrit centrifugue technique for the diagnosis of African trypanosomiasis. Acta Trop 1970;27:384-6.

19 Cattand P, Miezan BT, De Raadt P. Human African trypanosomiasis: use of double centrifugation of cerebrospinal fluid to detect trypanosomes. Bull World Health Organ $1988 ; 66: 83-6$
20 Noireau F, Lemesre JL, Nzoukoudi MY, Louembet MT, Gouteux JP, Frezil JL. Serodiagnosis of sleeping sickness in the Republic of the Congo: comparison of indirec immunofluorescent antibody test and card agglutination test. Trans $R$ Soc Trop Med Hyg $1988 ; 82: 237-40$

21 Kegels G, Criel B, van Lerberghe W, Mentens H, Magnus E, van Balen H. Screening for Trypanosoma brucei gambiense antibodies with the indirect fluorescent antibody test (IFAT). Ann Soc Belge Méd Trop 1992;72:271-81.

22 Papadopoulos MC, Abel PM, Agranoff D, Stich A, Tarelli E, Bell BA, et al. A novel and accurate diagnostic test for human African trypanosomiasis. Lancet 2004;363:1358-63.

23 Chappuis F, Loutan L, Simarro P, Lejon V, Buscher P. Options for field diagnosis of human African trypanosomiasis. Clin Microbiol Rev 2005;18:133-46.

24 Garcia A, Jamonneau V, Magnus E, Laveissiere C, Lejon V, N'Guessan P, et al. Follow-up of card agglutination trypanosomiasis test (CATT) positive but apparently aparasitaemic individuals in Cote d'Ivoire: evidence for a complex and heterogeneous population. Trop Med Int Health 2000;5:786-93.

25 Odiit M, Shaw A, Welburn SC, Fevre EM, Coleman PG, McDermott JJ. Assessing the patterns of health-seeking behaviour and awareness among sleeping-sickness patients in eastern Uganda. Ann Trop Med Parasitol 2004;98:339-48.

\author{
(Accepted 24 March 2006)
}

doi $10.1136 /$ bmj.38859.531354.7C

CUAMM-Angola and Angotrip Project, Caritas, Luanda, Angola

Walter O Inojosa medical officer

Inacio Augusto laboratory technician

Paulo M Abel medical coordinator

Centro per le Malattie Tropicali, Ospedale S Cuore, Negrar, Verona, Italy

Zeno Bisoffi doctor in chief

Instituto de Combate e Controlo das Tripanossomoiases, Ministério da Saúde, Luanda

Teofile Josenado director

Department of Tropical Medicine and Epidemic Control, Medical Mission Institute, Würzburg, Germany

August Stich head of clinical tropical medicine

Department of Infectious and Tropical Diseases, London School of Hygiene and Tropical Medicine, London WC1B 3DP

Christopher J M Whitty professor of international health

Correspondence to: W O Inojosa, Universidade Católica de Moçambique Ponta Gêa Rua Marquês de Soveral, 960 CP 821, Beira, Mozambique

inojosawalter@hotmail.com 